\title{
NIRCAM image simulations for NGST wavefront sensing
}

\section{Anand Sivaramakrishnan, Russell B. Makidon, Donald Frank Figer, Robert lan Jedrzejewski, Howard A. Bushouse, et al.}

Anand Sivaramakrishnan, Russell B. Makidon, Donald Frank Figer, Robert lan Jedrzejewski, Howard A. Bushouse, John E. Krist, H.S. Peter Stockman, Philip Hodge, Nadezhda M. Dencheva, Bernard J. Rauscher, Victoria G. Laidler, Catherine Mayumi Ohara, David C. Redding, Myungshin Im, Joel D. Offenberg, "NIRCAM image simulations for NGST wavefront sensing," Proc. SPIE 4850, IR Space Telescopes and Instruments, (5 March 2003); doi: $10.1117 / 12.461933$ Hawai'i, United States 


\title{
NIRCAM Image Simulations for NGST Wavefront Sensing
}

\author{
Anand Sivaramakrishnan ${ }^{a}$, Russell B. Makidon ${ }^{a}$, Donald F. Figer ${ }^{a}$, \\ Robert I. Jedrzejewski ${ }^{a}$, Howard A. Bushouse ${ }^{a}$, John E. Krist ${ }^{a}$, \\ H. S. Stockman ${ }^{a}$, Philip E. Hodge ${ }^{a}$, Nadezhda M. Dencheva ${ }^{a}$ \\ Bernard J. Rauscher ${ }^{a}$, Victoria G. Laidler ${ }^{b}$, Catherine Ohara ${ }^{c}$, \\ David C. Redding ${ }^{c}$, Myungshin $\operatorname{Im}^{d}$, and Joel D. Offenberg ${ }^{e}$. \\ ${ }^{a}$ Space Telescope Science Institute, \\ 3700 San Martin Drive, Baltimore, MD 21218, USA \\ ${ }^{b}$ Computer Sciences Corporation, \\ Space Telescope Science Institute, \\ 3700 San Martin Drive, Baltimore, MD 21218, USA \\ ${ }^{c}$ Jet Propulsion Laboratory, 4800 Oak Grove, Pasadena, CA 91109 \\ ${ }^{d}$ Infrared Processing and Analysis Center, \\ California Institute of Technology, Pasadena, CA 91125, USA \\ ${ }^{e}$ SSAI, GSFC, Greenbelt MD 20771, USA
}

\begin{abstract}
The Next Generation Space Telescope (NGST) will be a segmented, deployable, infrared-optimized 6.5m space telescope. Its active primary segments will be aligned, co-phased, and then fine-tuned in order to deliver image quality sufficient for the telescope's intended scientific goals. Wavefront sensing used to drive this tuning will come from the analysis of focussed and defocussed images taken with its near-IR science camera, NIRCAM. There is a pressing need to verify that this will be possible with the near-IR detectors that are still under development for NGST. We create simulated NIRCAM images to test the maintenance phase of this plan. Our simulations incorporate Poisson and electronics read noise, and are designed to be able to include various detector and electronics non-linearities. We present our first such simulation, using known or predicted properties of HAWAII HgCdTe focal plane array detectors. Detector effects characterized by the Independent Detector Testing Laboratory will be included as they become available. Simulating InSb detectors can also be done within this framework in future. We generate Point-Spread Functions (PSF's) for a segmented aperture geometry with various wavefront aberrations, and convolve this with typical galaxy backgrounds and stellar foregrounds. We then simulate up-the-ramp (MULTIACCUM in HST parlance) exposures with cosmic ray hits. We pass these images through the HST NICMOS 'CALNICA' calibration task to filter out cosmic ray hits. The final images are to be fed to wavefront sensing software, in order to find the ranges of exposure times, filter bandpass, defocus, and calibration star magnitude required to keep the NGST image within its specifications.
\end{abstract}

Keywords: NGST wavefront sensing and control, FPA detector simulation, cosmic ray removal, intra-pixel sensitivity

\section{INTRODUCTION}

The $6.5 \mathrm{~m}$ Next Generation Space Telescope (NGST) is required to use image-based wavefront sensing to maintain a Point-Spread Function (PSF) that possesses a Strehl ratio of $80 \%$ at a $20 \%$ bandwidth filter centered at $2 \mu \mathrm{m}$. There are also requirements on the shape and temporal stability of the PSF to enable cosmological origins studies to be carried out with the telescope. In order to meet this goal within its projected cost, NGST

Further author information: (Send correspondence to A.S.) 
wavefront sensing (WFS) is to use only imaging data, which will be obtained by the near-IR camera, NIRCAM, its primary imaging camera.

In order to ensure successful wavefront sensing, ground-based testbeds are being used to develop and refine WFS methods. The testbeds use CCD cameras to take imaging data, in order to keep development costs down while getting an early start on potential WFS problems that NGST might face. Given the fact that near-IR focal plane arrays (FPA's) typically have much poorer image quality than CCD detectors, there is a WFS credibility gap that can be bridged to some extent by simulations of FPA performance, given PSF calibration stars and the telescope geometry and optical quality. Since detectors which are to be used by NIRCAM will still be under development for some time, the exercise of simulating NGST's WFS images becomes an important one. As such detectors become available, they are tested by the Target Field Simulation Testbed (TFST) in the Independent Detector Testing Laboratory (IDTL), and their measured characteristics will be folded into the simulated WFS images to increase the veracity of the simulations, and improve confidence in NGST wavefront sensing and control (WFSC) methods $(1,2)$.

We outline the building blocks of our image simulations, and detail how we incorporate or plan to include detector effects, optical modelling, the NIRCAM calibration pipeline and the WFSC Executive software which will analyze NGST's WFS images to provide actuator commands to its active primary.

\section{WAVEFRONT SENSING AND CONTROL ON NGST}

NGST will be a segmented-mirror telescope, so its primary will have to be coarsely aligned and co-phased, and then fine-tuned. One segment can be chosen to be a reference segment. Other segments could then be aligned to this segment, and co-phased to it. The entire mirror will then be fine-phased. This paper deals with WFS during the last step, which should be a routine a 'figure maintenance' operation.

In a single maintenance visit described here, a set of already uplinked primary actuator commands (from a previous WFSC visit) is executed. Then images of a PSF calibration star are taken at different focus settings. The optical path delay (OPD) map of the pupil is recovered from these images using phase retrieval algorithms. This OPD is a measure of the aberration of the telescope pupil. The OPD is filtered to produce a set of actuator commands to be uplinked, and during the next WFSC maintenance visit, these commands are executed. Two visits can be placed back-to-back, and a wait placed between them to allow for on-ground data analysis to occur, or, during routine operations with NGST, the visits can take place at regularly-scheduled intervals.

\section{THE NEAR-INFRARED CAMERA (NIRCAM)}

NIRCAM is an imaging camera sensitive to near-IR (about $0.6-5.0 \mu \mathrm{m}$ ). We assumed a pixel scale of 0.0293 " per pixel. The instrument team for this camera has just been selected, but the detectors have not been chosen. The two detector material choices are $\mathrm{HgCdTe}$ and InSb. We implemented an HgCdTe-like detector in our simulations first simply because we could not develop simulations for both detectors at the same time. Our next step will be to implement the InSb detector simulation.

We assume 2048 x 2048 pixel FPA detector with a pixel readout rate of $100 \mathrm{kHz}$, though for reasons related to existing near-IR pipeline software, we restrict ourselves to calibrating $256 \times 256$ subarrays.

\subsection{NIRCAM Detector Readout}

Signal from the detector is assumed to be digitized with a 16 bit analog-to-digital converter (ADC).

The detector sits on a multiplexer (MUX). Each pixel is electrically connected to the MUX surface. The MUX is controlled by a few lines carrying control waveforms generated by the camera's electronics. The MUX performs reset and read operations at the behest of the control electronics situated in the controlling module located many meters away, behind NGST's sunshade. Current thinking is that analog output signals from the MUX will be relayed to an ADC that is located in this module, with approximately $6 \mathrm{~m}$ of cabling between the two.

The detector or a subset of the detector will be reset, either line-by-line or full-frame, to an externally supplied RESET voltage. The pixels are essentially capacitors which get charged (to up to a full-well maximum 
of $\sim 30,000 \mathrm{e}$ ) when light hits the detector. Hardware registers on the MUX near the edges of the detector connect a single pixel to an output source-follower amplifier (depending on the location of a single high bit in the registers). After a time interval comparable to or greater than the time constant which characterizes the decay of transients in the output analog signal line, the ADC samples the pixel voltage. This sampling is non-destructive in that it does not affect the charge in the pixel.

Usually the high bit in the 'fast' register is then moved one position along by a control signal to connect the next pixel in the same row to the output amp. This process is repeated as many times as desired, to read some or all of the pixels in a row. To read from the next row, the high bit in the 'slow' register is bumped one location up by a control signal. The settling time is determined by the effective RC time constant of the electrical connection to the ADC. This is expected to be primarily due to cables, though MUX and pixel capacitances could also be a contributing factor. Both NGST NIRCAM detector candidates have similar readout schemes. The number of output amps, the exact readout patterns, and the pixel rate depend on the manufacturer.

The process of simulating the detector is central to the image simulations. Since in-focus or near-focus PSF calibration stars are very high contrast images, the issue of electronic settling time is important. We wrote an Amp object that retains temporal history, and implements an RC-like exponential decay time of its output voltage given the time of its previous read and its output voltage at the time the FET's switch the amp's connection to the next pixel. We use a time constant of 440ns for the settling time, based on laboratory measurements on a typical near-IR MUX. The IDTL cabling has a very much shorter time constant than the MUX itself. The 440ns time constant is an unrealistically low figure for NGST if one is to assume the MUX is to be connected to its ADC by $6 \mathrm{~m}$ of $12 \mathrm{pF}$ /foot cable possessing $\sim 2 \mathrm{k} \Omega$ total resistance, for instance. Such a configuration would possess a net $\mathrm{RC}$ time constant of the order of $1 \mu \mathrm{s}$.

For this version we use only a single output amp, though it is possible to simulate multiple output amps and their temporally interleaved operation using the existing structures, should that become relevant in future simulations.

\subsection{Detector and electronic non-linearities}

Experience with ground- and space-based near-IR detectors indicates that the following detector effects will have to be understood in order to determine how best to control and calibrate NIRCAM detectors for WFSC (as well as science).

- Wavelength and temperature dependent flat-fields

- Detector non-linearity

- Latent charge effects (persistence from bright stars, cosmic rays)

- Electronic crosstalk from other activity

- Voltage drifts (e.g. 1/f noise)

- Residual bias drifts ('shading')

- Intra-pixel sensitivity

- Pixel crosstalk due to electronics settling time

- Amp glow from on-chip outputs

- Fringing (Newton's rings in the detector material, especially in narrow band filters)

- Up-the-ramp cosmic ray filtering

Our simulations are structured to allow us to model any of these effects if we feel they require quantitative estimation. We keep track of times of individual pixel reads inside our code, and the number of output amps used, along with their temporal voltage history. This enables complex effects to be simulated if there is a perceived need to simulate them. For example, latent charge effects are implementable with data files with the charge distribution on the chip, along with temporal information. 


\subsection{Filters and grisms}

Science drivers for NGST require broad band filters. The inverse fractional bandwidth, $\lambda / \Delta \lambda_{F W H M}$, is referred to as the resolving power of the filter, or its R-value (the central wavelength is $\lambda$, and $\Delta \lambda_{F W H M}$ is the full-width at half maximum of the filter transmission curve). R-values of 3 to 5 will be common in the NGST filter set. WFS from images requires narrower filters than these typical science filters. R-values of $\sim 40$ might be needed for inspection of in-focus images out to a spatial frequency of approximately $2 D / R=D / 20$ on the primary $(D$ being the diameter of the primary mirror). Experience with HST NICMOS indicates that such bandwidths are required to examine small structures in its pupil using near-IR images (3).

Defocusing elements might also be needed in the filter wheels to provide 'focus diversity' without moving the telescope secondary mirror. An in-focus image cannot produce a unique OPD on analysis: the sign of the aberration cannot be determined from this data alone. Specialized optical elements in the NIRCAM optics could be an essential part of the WFSC system.

\subsection{Exposure patterns and readout timing}

Infrared detectors are more non-linear and unstable than CCD's. Significant improvements in detector quality have occurred since the HST NICMOS detectors, but some of the NICMOS detector nonlinearities are likely to exist in NGST's detectors (if the HgCdTe material is chosen). Both detectors will possess their own characteristics, such as the decay timescale of image latency $(4,1)$, temperature dependence and so on.

Exposure patterns will be tailored to the detector characteristics. The temporal pattern we use might be more suited to $\mathrm{HgCdTe}$ detectors, but we can tailor our simulation to account for read patterns which differ from the one described below.

We simulate an evenly-spaced (in time) set of non-destructive reads occurring after a full-chip reset. We encapsulate the individual read time offsets of each pixel from the start of a chip or subraster read by creating a numerical array of offset times which are added to the nominal time of the read. The latter is the time the first pixel of the chip is sampled. Our assumption of a $100 \mathrm{kHz}$ pixel sampling rate leads to a 10 microsecond offset in the time two successive pixel reads are performed by the ADC. We implement the multiple non-destructive reads using a list of readout times, so other read patterns (e.g., Fowler sampling) can be implemented without writing any new code. The flexibility of assigning pixel offset times with an input array will enable electronic crosstalk to be modelled later if it becomes necessary, using multiple Amp objects on a detector.

The multiple reads enable cosmic ray rejection by data processing pipelines. Dark frames for most or all exposure times utilized will be required to calibrate the data because of non-linear dark current behavior and reset transients. Detector temperatures depend somewhat on the readout and reset history of the detector. Dark current can be simulated in the multiple read simulation, as can the detector response non-linearity (which is fairly gentle over most of the useful range of accumulated charge). We can also accumulate real dark frames along with simulated sky counts. Similarly, we can use real read noise files instead of simulated ones.

\section{TELESCOPE SIMULATION}

In order to follow the laboratory demonstrations of wavefront sensing and control that are being developed on the NGST Wavefront Control Testbeds (WCT), for the first set of images we used the three-segment WCT-2 aperture geometry. This is a set of three circular apertures that enable WFSC methods on separate segments to be proved. The aperture is shown in Figure (1).

\subsection{Monochromatic PSF}

The optical path delay (OPD) map for the pupil is generated by using a first order expansion around perfectly aligned and phased segments. There are nine variables describing the aberrations of the WCT-2 pupil: tip, tilt and piston on each of the three segments. Pupil shape changes (clocking of the pattern or individual segments, and lateral shifts) will require separate pupil support functions if they are to be modelled. By selecting a realization of these nine variables, a constant 'gain' matrix relating these to the OPD map is used to generate the OPD map which results from any combination of these variables. We use the JPL-developed MACOS 
optical modelling software to create the 'linearized MACOS model' of the optical system, which is a floating point $9 \mathrm{xN}$ matrix, where $\mathrm{N}$ is the number of samples in the numeric array defining the OPD. This approach can be generalized to other pupil shapes, and more degrees of control, as would be appropriate for a full-up simulation of the NGST primary mirror segments and their degrees of control. There would be some threshold of the input variables' magnitudes where this linear approximation breaks down, but we expect the linearized MACOS model to be useful for fine-phasing simulations of NGST.

\subsection{Polychromatic PSF}

We pad the pupil array by the required number of zero-filled samples to create identical physical pixel scales at different wavelengths. The input variables required for this task are the following:

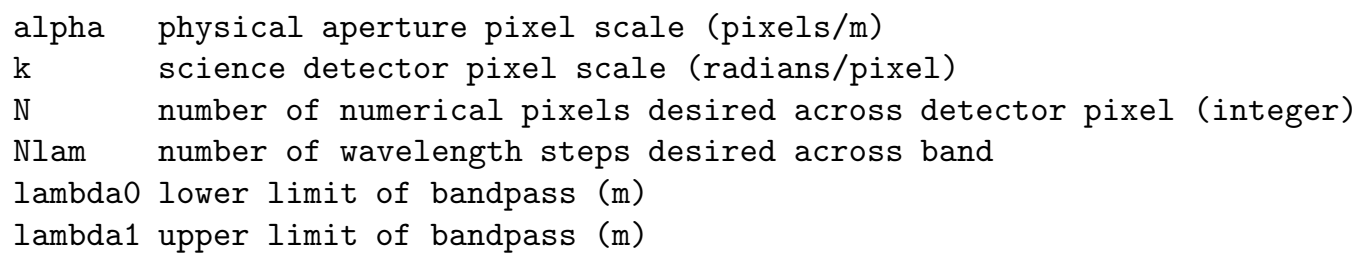

The number of 'numerical' pixels desired across a detector pixel is a sub-pixellation which must be done in order to model intra-pixel sensitivity (IPS).

Applying a fast Fourier transform (FFT) to the resultant arrays produces images that can be stacked pixelfor-pixel if the zero-frequency pixel (the image center, physically speaking) is aligned. We trim the image to the smallest sized array in the stack, which is at the shortest wavelength. We also ensure that the FFT arrays have an even-number of samples across a side. We weight the individual image frames evenly, though that could be changed to match any desired target spectrum. This even weighting is equivalent to a flat spectrum in photons per second per wavelength interval. The final polychromatic PSF is normalized to contain a total power of unity. We chose the FFTW (5) library because of its ability to perform FFTs of arbitrary size (as opposed to restricting the array size to be a power of 2 ). We use a Python-wrapped version of this software known as fftw-numpy (6).

\section{ASTRONOMICAL SOURCES}

\subsection{Background galaxies}

We generate background galaxies to populate our field of view using the methods in (7). We assume a clear area corresponding to an unobstructed disk of $6.5 \mathrm{~m}$ diameter, and a total throughput of 0.63 to create a perfectlysampled sky brightness pattern with elliptical, spiral, S0 and irregular galaxies, in the Kab filter bandpass. We refer the reader to the quoted reference for details on the cosmology used, the fractions of the various kinds of galaxies generated, and so on. We resample this galaxy background into $3 \times 3$ 'sub-pixels', in order to incorporate the effects of IPS of the detector on WFSC. Since galaxy structure is unlikely to contain significant power on scales as small as a single detector pixel, we do not generate the galaxy images themselves on this finer IPS scale (although this is in principle possible to do, but is very computationally intensive). We can change the resampling factor used to match the fineness of the grid of IPS measurement in the lab. For HST NICMOS the corner sub-pixels were reported to be as much as $30 \%$ less sensitive than the pixel centers (8). We expect NGST detectors to have a more even sensitivity across their pixels. We use a moderately pessimistic model of the corner sub-pixels being $80 \%$ as sensitive as the central sub-pixel, and the middle of the edges of the pixel being $90 \%$ as sensitive as the center. 


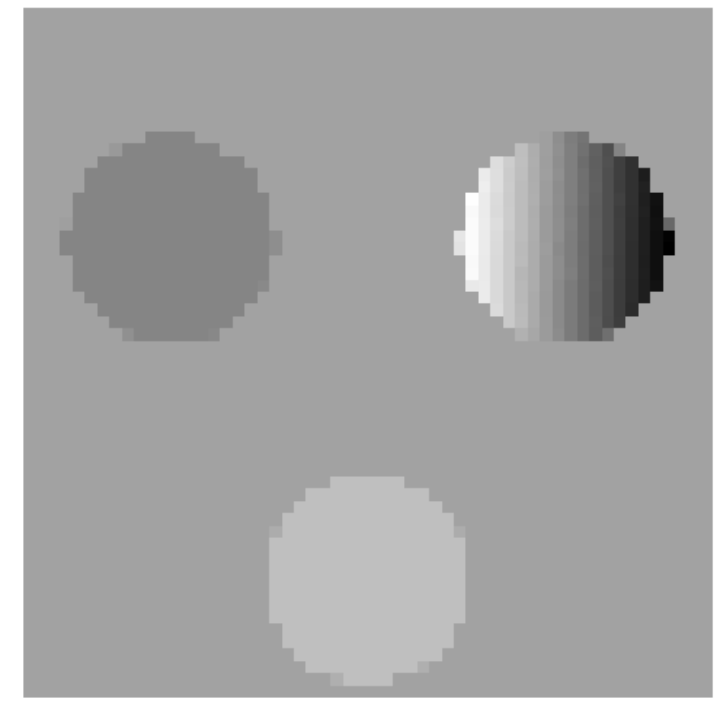

Figure 1. The WCT-2 three circular segment pupil shape. A pupil of this shape, scaled up to the equivalent of a $6.5 \mathrm{~m}$ diameter unobstructed aperture telescope's collecting area is used in these simulations. Other pupil shapes with finer spatial sampling can be used instead. The pupil sampling sets the scale of the PSF images generated. These pupils were 49 pixels across at their widest horizontal extent in the numerical array. In this realization the top left segment has pure piston of $200 \mathrm{~nm}$, and the top right segment has an X-tilt which puts its leftmost pixel at a height of 336nm, and its right edge at $-457 \mathrm{~nm}$. The bottom segment has zero piston, tip, and tilt. All figures are optical path delay (OPD) rather than surface height.
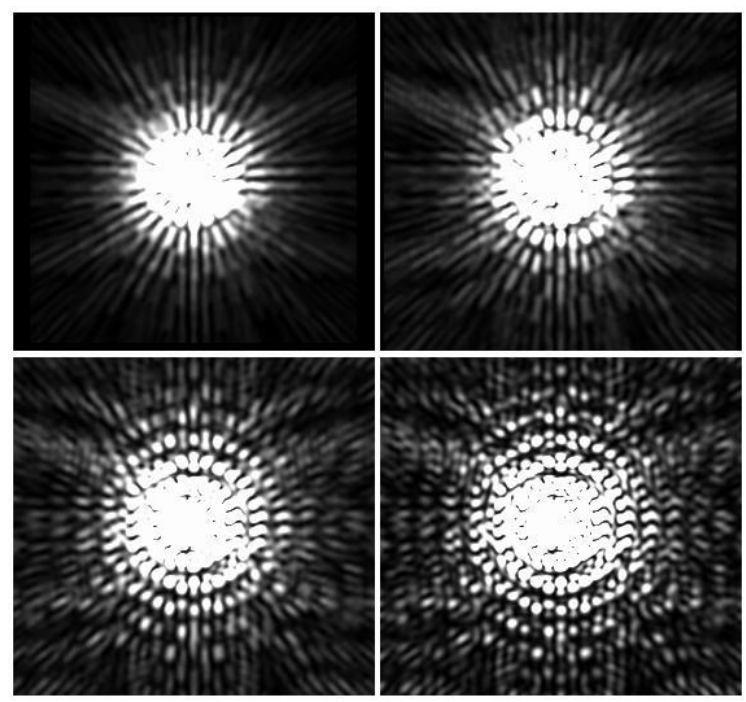

Figure 2. PSFs from the WCT-2 three circular segment aperture using the OPD map shown in Figure 1. Polychromatic PSFs with R-values of 3, 5, 10 and 20, in (left-right, top-bottom order) shown on the same logarithmic stretch. The pixel scale in these images is three pixels across the assumed physical detector pixel (which has an angular size of 0.0293 arcseconds), because we use a $3 \times 3$ subsampling of the pixel in order to model intra-pixel sensitivity. The top right PSF is used in the image simulation shown in Figure 3. 

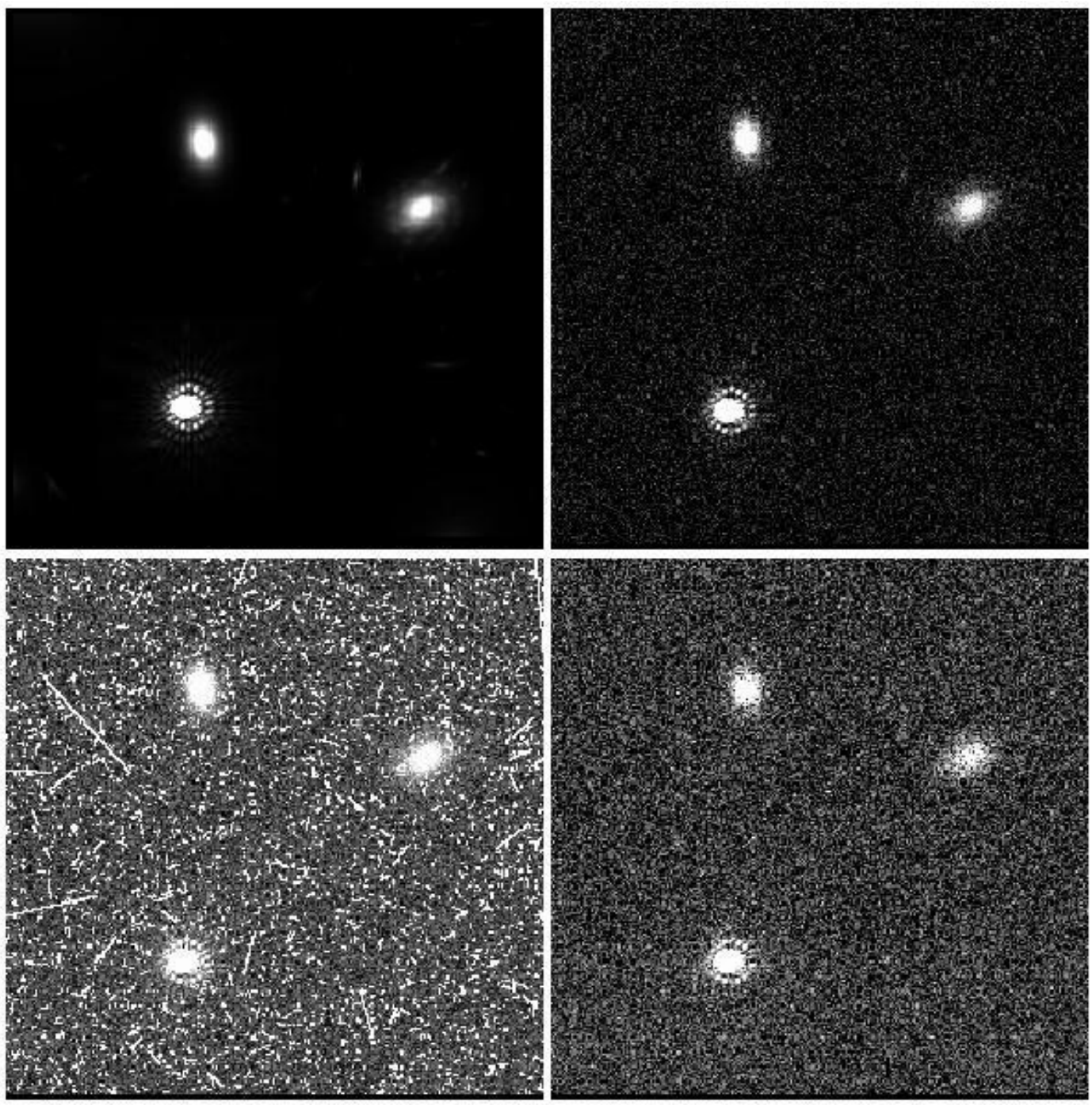

Figure 3. Simulated $K_{A B}$-band images with a $6.5 \mathrm{~m}$ diameter collecting area telescope (all on the same linear stretch). The PSF used corresponds to the WCT-2 aperture with $7.2 \mathrm{~m}$ across its largest diameter. The pixel scale is 0.0293 arcseconds. A $256 \times 256$ pixel region is shown $\left(7.5 \times 7.5\right.$ arcseconds). The lower left object is a $K_{A B}=19$ star. One might expect to find approximately one such star in a 1 arcminute field of view chosen at random, away from the galactic plane, though this number is still subject to considerable uncertainty. The other two objects are simulated background galaxies. These images contain many more galaxies than are visible at this stretch. Top left: PSF-convolved, but no Poisson noise. Top right: PSF-convolved, with Poisson noise. Bottom left: PSF-convolved, with Poisson noise and cosmic rays (1hour exposure). Bottom right: The 6-read 1 hour exposure calibrated with HST NICMOS's CALNICA calibration task to remove cosmic rays (see text). 


\subsection{Stars}

We query the version of the Space Telescope Guide Star II catalog* with a pointing on the sky to obtain a list of all stars in that catalog which would be present on the detector image. We do not go further than the chip boundaries in recording stellar locations, though this feature may benefit precision photometric studies using our software because of mirror scatter (9). We assume every star found is a G0 star with a $V-K$ color of 1.41 magnitudes (10). If the stellar spectral type is known, it is an easy task to use a magnitude and a color to obtain a better estimate of the $K a b$ magnitude of the star. We find that in typical pointings there are no stars from this catalog on a 1 arc minute square field of view. When we use pointings near clusters we find as many as half a dozen targets from the GSCII catalog on the detector. The image we display contains an artificially-injected $K a b=19$ star in it.

\subsection{Zodiacal light}

We have not included the effect of zodiacal light in these simulations because it probably has a small effect on the bright star images expected during NGST's WFS data acquisition. Since the zodiacal light is expected to be smoothly distributed across the detector, it would be easy to incorporate this source of background into the sky photon rate input file by addition of an image corresponding to the zodiacal background. We do intend to add this background source into our simulations at some point in the future. Zodiacal light might prevent routine science images from being used for 'in-focus PSF optimization' (IPO).

\subsection{Telescope thermal background}

We have not included the effect of thermal radiation from NGST optics and structures. At wavelengths shorter than about $1.8 \mu \mathrm{m}$ (where WFS is more effective because the same OPD produces larger phase aberrations), thermal background on HST is not significant (11). We therefore do not expect thermal background to be a significant factor in WFSC on NGST at around $2 \mu \mathrm{m}$. If we had to model mid-IR instrument WFS, we would need to estimate this contribution to the background. We have a straightforward software development path to this module, because we can use existing code or the object design developed for the HST NICMOS exposure time calculator $(12,13)$.

\section{COSMIC RAYS}

We use the code described in (14), which we modify to use a solid body pixel model $20 \times 20 \times 10 \mu \mathrm{m}$ in size. We use its default cosmic ray (CR) event rate of 4 per square $\mathrm{cm}$ per second. Following the example in (14), we use its default values of $100 \pm 10$ electrons per $0.1 \mu \mathrm{m}$ of travel within the detector, with $10 \%$ of the CR's being He nuclei with four times the electron liberation rate just quoted. We do not include the effects of a large sun shield, though there is an option to set the sun shield size in the code. Similarly, CR events can be made to spread liberated electrons into nearest neighbor and second-nearest neighbouring pixels. We do not switch this feature on for the simulations shown in Figure 3.

We generate realizations of $\mathrm{CR}$ electron counts in 6 up-the-ramp non-destructive reads, with a time difference of 720 seconds between reads. This 1-hour exposure with a zeroth read and five more reads is our test exposure pattern. This might be a typical single NGST science exposure, with several of these exposures performed back-to-back at the same pointing during a visit to one target.

${ }^{*}$ The Guide Star Catalogue II is a joint project of the Space Telescope Science Institute (STScI) and the Osservatorio Astronomico di Torino. STScI is operated by the Association of Universities for Research in Astronomy (AURA), for the National Aeronautics and Space Administration (NASA) under contract NAS5-26555. The participation of the Osservatorio Astronomico di Torino is supported by the Italian Council for Research in Astronomy. Additional support is provided by European Southern Observatory, Space Telescope European Coordinating Facility, the International GEMINI project and the European Space Agency Astrophysics Division. 


\subsection{Cosmic ray rejection}

We place 256 x 256 pixel frames of simulated up-the-ramp reads into a multi-extension FITS file with the same structure as that of the HST NICMOS pipeline data, and process it using CALNICA (15), the HST NICMOS calibration program which transforms raw HST NICMOS data frames into a countrate frame. We run this pipeline within the STSDAS ${ }^{\dagger} / \mathrm{IRAF}^{\ddagger}$ environment.

The cosmic ray rejection algorithm fits a straight line to the counts-exposure time graph of a single pixel, and detects any readout that jumped more than a user-selected number of standard deviations above the line. A noise file for each read must be created: we use the MKNOISE task in IRAF to create realizations of each frame with Poisson noise, and Gaussian-distributed read noise frames with a 15 electron standard deviation. These noise files are packaged with the raw input data frames that are fed to CALNICA. Further details on the cosmic ray rejection algorithm can be found in CALNICA help files and on-line documentation for the HST NICMOS camera. We can substitute real read noise files for the simulated ones when we model any particular detector, by adding single data frames of read noise to each simulated read, and subtracting a many-frame average of read noise from the data in the calibration step.

\subsection{Cosmic ray and image persistence}

Cosmic ray persistence is a longer term issue we would like to address. It is more troubling than the CR-removal issue. HST NICMOS detectors' behavior after passage through the South Atlantic Anomaly reveal that time is the only cure for charge persistence (16). The higher CR event rates of NGST's circum-L2 orbit will cause a contribution to the noise background which will need modelling at some time in order to verify that both faint-object science and WFS can be performed satisfactorily. We could use the same cosmic ray simulation code to study this effect in future, but we do not model CR persistence at the moment.

Image persistence is similar in nature to CR persistence: WFS targets will need to be placed on areas of the detector which have not had bright objects on them immediately before the observation. Bright star WFS observations might also need to be done on different areas of the chip during one visit. We will be able to model this effect when we feel it is important to understand it quantitatively.

\section{SOFTWARE ORGANIZATION AND INTERFACES, AND FUTURE WORK}

We re-use software written in FORTRAN, C, Python, MATLAB, $\mathrm{C}^{++}$, as well as entire packages such as IRAF, STSDAS, PyRAF (17), PyFITS ${ }^{\S}$, and FFTW (5). In order to simplify the interfaces, we transfer data between the various components in the form of FITS files and saved scripts running different tasks. All new code was written in Python. This approach has enabled us to reach the stage of creating a simulated calibrated image rapidly. What remains to be done from the software point of view is to enable various modifications discussed above as they become necessary, and to write a simple user's guide with a developer's section. We do not plan to integrate the entire task on one computer or in one language. The principle goal of our exercise is to discover the limitations of various wavefront sensing approaches and help find some resolution for these. Our first target is to determine the required filter bandwidth of the fine-phasing WFSC images, as discussed in section 3.3. The next one will probably be the degree of defocus required to accomplish routine PSF maintenance. Other uses for this software include simulating fine guidance sensor images with assumed or measured detector and electronics characteristics. This software can also be re-used to model mid-IR detectors for NGST, though a telescope thermal background module or data file will have to be written for that purpose.

\section{ACKNOWLEDGMENTS}

We acknowledge useful discussions with Eddie Bergeron, Perry Greenfield, Todd Miller, Megan Sosey, and Rick White.

\footnotetext{
${ }^{\dagger}$ STSDAS is distributed by STScI, which is operated by AURA Inc. under contract to NASA.

${ }^{\ddagger}$ IRAF is distributed by NOAO, which is operated by AURA Inc. under contract to the NSF.

${ }^{\S}$ http://www.adass.org/adass/proceedings/adass99/P1-55/
} 


\section{REFERENCES}

1. D. F. Figer, M. Agronin, J. Balleza, R. Barkhouser, L. Bergeron, G. R. Greene, S. R. McCandliss, B. J. Rauscher, T. Reeves, M. W. Regan, U. Sharma, and H. S. Stockman, "The I detector Testing Laboratory and the NGST detector program," in IR Space Telescopes and Instruments, J. Mather, ed., Proc. SPIE $4850,2002$.

2. U. Sharma, D. F. Figer, A. Sivaramakrishnan, M. Agronin, J. Balleza, R. Barkhouser, L. Bergeron, G. R. Greene, S. R. McCandliss, B. J. Rauscher, T. Reeves, M. W. Regan, and H. S. Stockman, "Intra-pixel sensitivity in NIR detectors for NGST," in IR Space Telescopes and Instruments, J. Mather, ed., Proc. SPIE 4850, 2002.

3. J. E. Krist, D. A. Golimowski, D. Schroeder, and T. J. Henry, "Characterization of NICMOS camera 2 PSFs," PASP 110, pp. 1046-1058, 2000.

4. S. L. Solomon, Near Infrared Spectroscopic Imaging of NGC 7023 and Charge Trapping in InSb Photodiode Arrays, PhD Thesis, University of Rochester, 1998.

5. M. Frigo and S. G. Johnson, "The Fastest Fourier Transform in the West," in Technical Report MIT-LCSTR-728, Massachusetts Institute of Technology, 1997.

6. T. Oliphant, "Private communication," 2000.

7. M. Im and H. S. Stockman, "A simulation of the NGST deep field," in Astrophysics with Infrared Surveys: A Prelude to SIRTF, M. D. Bicay, R. M. Cutri, and B. F. Madore, eds., ASP Conference Ser. 177, p. 75, 1999.

8. T. R. Lauer, "The photometry of undersampled point-spread functions," PASP 111, p. 1434, 1999.

9. J. R. Kuhn and S. L. Hawley, "Some astronomical performance advantages of off-axis telescopes," PASP 111, pp. 601-620, 1999.

10. J. R. Kenyon and S. L. Hartmann, "Pre-main-sequence evolution in the Taurus-Auriga molecular cloud," ApJS 101, p. 117, 1995.

11. M. Robberto, A. Sivaramakrishnan, J. J. Bacinski, D. Calzetti, J. E. Krist, J. W. MacKenty, J. Piquero, and M. Stiavelli, "Performance of HST as an infrared telescope," in UV, Optical and IR Space Telescopes and Instruments, J. B. Breckinridge and P. Jacobsen, eds., Proc. SPIE 4013, 2000.

12. A. Sivaramakrishnan, B. Simon, S. Holfeltz, H. Shukla, and M. Sosey, "The new NICMOS exposure time calculator: software and installation," in STScI Instrument Science Reports: NICMOS ISR-00-005, Space Telesecope Science Institute, 2000.

13. A. Sivaramakrishnan, S. Holfeltz, B. Simon, and M. Robberto, "The NICMOS exposure time calculator: algorithms and user interface," in STScI Instrument Science Reports: NICMOS ISR-00-001, Space Telesecope Science Institute, 2000.

14. J. D. Offenberg, R. J. Hanisch, D. J. Fixsen, H. S. Stockman, M. A. Nieto-Santisteban, R. Sengupta, and J. C. Mather, "Multi-readout data simulator," LANL Archives arXiv:astro-ph/0005403, 2000.

15. H. A. Bushouse, C. Skinner, and J.MacKenty, "The STScI NICMOS pipeline CALNICA, single image reduction," in STSCI Instrument Science Reports: NICMOS ISR-97-029, Space Telesecope Science Institute, 1997.

16. J. R. Najita, M. Dickinson, and S. Holfeltz, "Cosmic ray persistence in NICMOS data," in STScI Instrument Science Reports: NICMOS ISR-98-001, Space Telesecope Science Institute, 1998.

17. R. L. White and P. Greenfield, "Using Python to modernize astronomical software," in 8th International Python Conference Proceedings, D. Beazley, ed., Foretec Seminars, 2000. 